A bottom-up approach for controlled deformation of carbon nanotubes through blistering of supporting substrate surface 


\title{
A bottom-up approach for controlled deformation of carbon nanotubes through blistering of supporting substrate surface
}

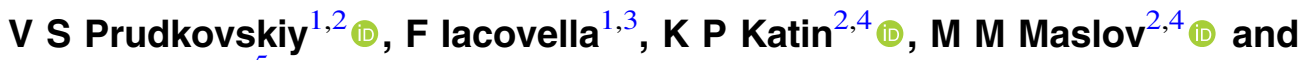 \\ N Cherkashin ${ }^{5}$ (]) \\ ${ }^{1}$ Department of Physics, University of Crete, Heraklion, 71003, Greece \\ ${ }^{2}$ Laboratory of Computational Design of Nanostructures, Nanodevices and Nanotechnologies, Research \\ Institute for the Development of Scientific and Educational Potential of Youth, Aviatorov Street 14/55, \\ Moscow 119620, Russia \\ ${ }^{3}$ Foundation for Research and Technology, IESL, Heraklion, 71110, Greece \\ ${ }^{4}$ Department of Condensed Matter Physics, National Research Nuclear University 'MEPhI', Kashirskoe \\ Shosse 31, Moscow 115409, Russia \\ ${ }^{5}$ CEMES-CNRS and Universite de Toulouse, 29 rue J. Marvig, F-31055 Toulouse, France
}

E-mail: vladimir.prudkovskiy@gmail.com

\begin{abstract}
Tuning the band structure and, in particular, gap opening in 1D and 2D materials through their deformation is a promising approach for their application in modern semiconductor devices. However, there is an essential breach between existing laboratory scale methods applied for deformation of low-dimensional materials and the needs of large-scale production. In this work, we propose a novel method which is potentially well compatible with high end technological applications: single-walled carbon nanotubes (SWCNTs) first deposited on the flat surface of a supporting wafer, which has been pre-implanted with $\mathrm{H}^{+}$and $\mathrm{He}^{+}$ions, are deformed in a controlled and repetitive manner over blisters formed after subsequent thermal annealing. By using resonant Raman spectroscopy, we demonstrate that the SWCNTs clamped by metallic stripes at their ends are deformed over blisters to an average tensile strain of $0.15 \pm 0.03 \%$, which is found to be in a good agreement with the value calculated taking into account blister's dimensions. The principle of the technique may be applied to other $1 \mathrm{D}$ and $2 \mathrm{D}$ materials in perspective.
\end{abstract}

Keywords: carbon nanotubes, strain, Raman spectroscopy, blisters, ion implantation, nanofabrication

(Some figures may appear in colour only in the online journal)

\section{Introduction}

Extremely high mobility of charge carriers [1, 2], presence of ballistic transport [3] and complementary metal-oxide-semiconductor compatibility of nanocarbon materials [4] makes them promising candidates for replacing $\mathrm{Si}$ in channels of high frequency transistors [5-7]. However, graphene and metallic single-walled carbon nanotubes (SWCNTs) are zerobandgap materials $[8,9]$ that hamper their direct application in the high frequency nanoelectronics. Particularly for
SWCNTs, several preselection methods have been developed [10-15] in order to exclude metallic SWCNT and collect only semiconducting ones for following device nanofabrication. Nevertheless, no one solves this problem completely, as a resulting semiconducting purity of selected SWCNTs is always under $100 \%$ and the cost of such preselection is extremely high. Recently, it was demonstrated that bending of graphene and metallic SWCNTs leads to the bandgap opening [16-20]. The essential drawback of the approaches used to bend these materials is the absence of the control over their 
deformation and the poor compatibility of laboratory scale methods with the needs of large-scale production. The development of a controlled and repetitive bending of graphene and SWCNTs over meter-scale areas would pave the way to a sophisticated manipulation of their electronic band structure and thus to their direct application in modern high frequency semiconductor devices. To achieve this goal, the development of new approaches to strain engineering of graphene and SWCNTs are essential.

In this work, we demonstrate a novel method for the bottom-up deformation of SWCNTs initially deposited on the flat surface of a supporting wafer which has been preimplanted with $\mathrm{H}^{+}$and $\mathrm{He}^{+}$ions. The transfer of the strain to SWCNTs is realized through wafer surface blistering which appears after thermal annealing.

Blistering is a phenomenon which was observed in $\mathrm{Si}$, $\mathrm{SiC}$ and many other materials after $\mathrm{H}^{+}$or $\mathrm{He}^{+}$ions implantation for a long time [21-24]. When $\mathrm{H}^{+}$ions are implanted in a Si substrate, a depth location of a Gaussianlike hydrogen atoms distribution is defined by ions implantation energy. During annealing, the hydrogen atoms and some of the vacancies generated by implantation coprecipitate and form two-dimensional circular objects named platelets $[25,26]$. These platelets, filled with $\mathrm{H}_{2}$ gas, grow in size during annealing until they become large enough and elastically interact and finally coalesce to form micro-cracks distributed within a thin layer at a certain depth from the wafer surface [23]. When these micro-cracks are close to the free surface of the wafer, the stress generated onto the silicon matrix by the pressure inside the micro-cracks can elastically relax through the deformation of the surface, i.e. the formation of protuberances named blisters $[27,28]$. When $\mathrm{H}^{+}$and $\mathrm{He}^{+}$ions are co-implanted, helium incorporates and over-pressurizes the hydrogen platelets during annealing and thus promotes their more effective mechanical coalescence and formation of blisters [24, 29].

The density, the height and the lateral dimensions of blisters can be varied independently one from the other using different: (i) temperature and time of annealing [23]; (ii) $\mathrm{He}$ and $\mathrm{H}$ fluences [24]; (iii) He to $\mathrm{H}$ fluences ratio [29, 30]; (iv) $\mathrm{He}$ and $\mathrm{H}$ relative depth distributions (imposed by respective ions energies) [31]; (v) $\mathrm{He}$ and $\mathrm{H}$ relative order of implantation [32] and, eventually, (vi) depth position of the crack nucleation and propagation (imposed by $\mathrm{H}$ ions implantation energy) [24]. By choosing an appropriate combination of all implantation and annealing conditions, one would expect to achieve the required density, surface fraction, average diameter and height-to-diameter ratio of the blisters.

In this work, by using resonant Raman spectroscopy as an independent probe for strain measurement [33], we compare the effect of blistering on SWCNTs being either free standing or clamped by metal stripes at their edges. Finally, we will discuss the impact of the blisters' diameters and heights on the strain generated within SWCNTs.

\section{Experimental section}

(001) $\mathrm{Si}$ wafer covered by a $25 \mathrm{~nm}$ thick thermally grown $\mathrm{SiO}_{2}$ layer was co-implanted at room temperature by $\mathrm{He}^{+}$ions at $8 \mathrm{keV}$ then $\mathrm{H}^{+}$ions at $3 \mathrm{keV} \mathrm{H}^{+}$with the same fluences of $\mathrm{He}$ and $\mathrm{H}$ ions, each one of $1.2 \times 10^{16} \mathrm{~cm}^{-2}$. Pre-selected $99 \%$ semiconducting arc discharge SWCNTs 'IsoNanotubes-S ${ }^{\mathrm{TM}}$, from 'NanoIntegris' company were deposited from aqueous surfactant solution on the substrate. We use the pre-selected semiconducting SWCNTs with a narrow diameter distribution in order to obtain homogeneous single-type Raman spectrum over a large surface and minimize spectrum variations related to a difference in the chirality of the tubes. Concentration of SWCNTs was adjusted in order to have individual nanotubes spread over the surface avoiding bundling and clustering. Diameter of the nanotubes ranges from 1.2 to $1.7 \mathrm{~nm}$ and length from 0.3 to $5 \mu \mathrm{m}$. Some of SWCNTs were clamped by metallic stripes in order to prevent sliding along the surface during strain application (figure 1). Metallic stripes were fabricated by means of conventional electron beam lithography using polymethyl methacrylate (PMMA) resist, followed by metallization and liftoff process. Stripes were spaced by $0.5,1,2,3$ and $4 \mu \mathrm{m}$.

The sample was annealed at $550{ }^{\circ} \mathrm{C}$ in nitrogen gas for 30 min resulting in a blistering of the surface. The blisters' morphology has been characterized by atomic force microscopy (AFM), scanning electron microscopy and optical microscopy measurements. The average blister diameter and height are found to be of about $0.7 \mu \mathrm{m}$ and $15 \mathrm{~nm}$, respectively. The detailed characterization of the blisters' morphology can be found elsewhere [24]. The amount of strain in SWCNTs was monitored by resonant Raman spectroscopy prior and after substrate surface blistering. Raman spectra were acquired using the excitation wavelength of $473 \mathrm{~nm}$ in the back-scattering configuration with $100 \times$ objective, which corresponds to a $\sim 1 \mu \mathrm{m}$ laser spot size. The laser power was kept to $1 \mathrm{~mW}$ with an accumulation time of $20 \mathrm{~s}$.

\section{Results and discussion}

Figure 2(a) shows the spectra obtained on the non-clamped semiconducting SWCNTs (zone A in figure 1(c)) before (black line) and after (red line) surface blistering. Both of the spectra show several characteristic signatures of SWCNTs: radial breathing mode $(\mathrm{RBM}), \mathrm{D}, \mathrm{G}^{-}, \mathrm{G}^{+}$and $2 \mathrm{D}$ Raman bands [34]. Blistering did not lead to any considerable position shift of any of these peaks. The same behavior is observed for the spectra obtained on the SWCNTs either clamped only at one end or clamped at both ends in between metallic stripes spaced by a distance of more than $2 \mu \mathrm{m}$. These facts indicate that the non (or partially) clamped SWCNTs are not (or weakly) strained after blistering. Possible sliding of SWCNTs under stress over the $\mathrm{SiO}_{2}$ surface would indicate that their stiffness exceeds van-der-Waals forces which are responsible for their adhesion to the $\mathrm{SiO}_{2}$ surface [35]. 
a)

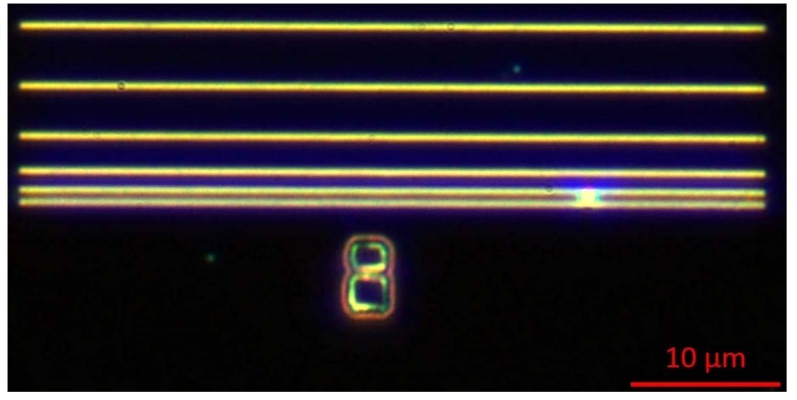

c)

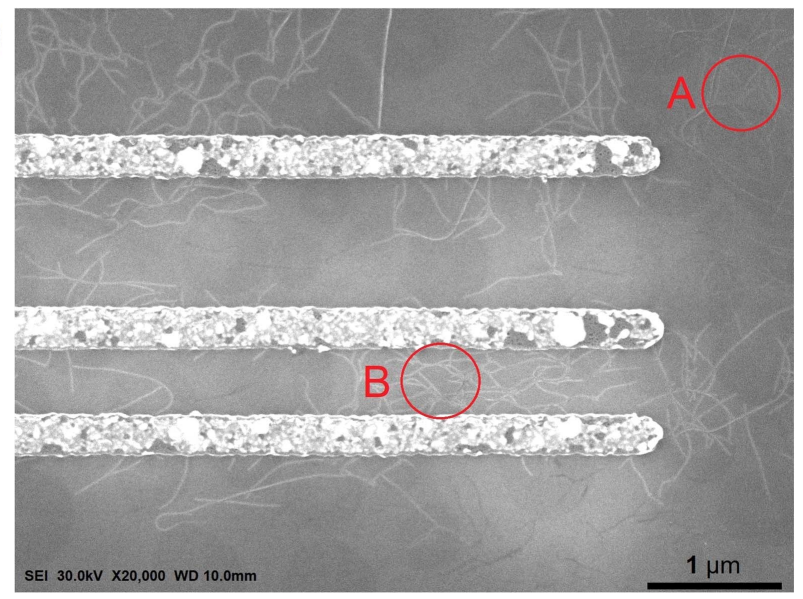

b)

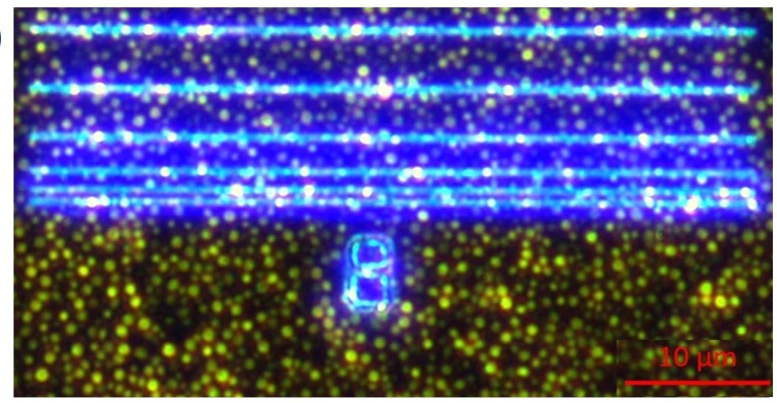

d)

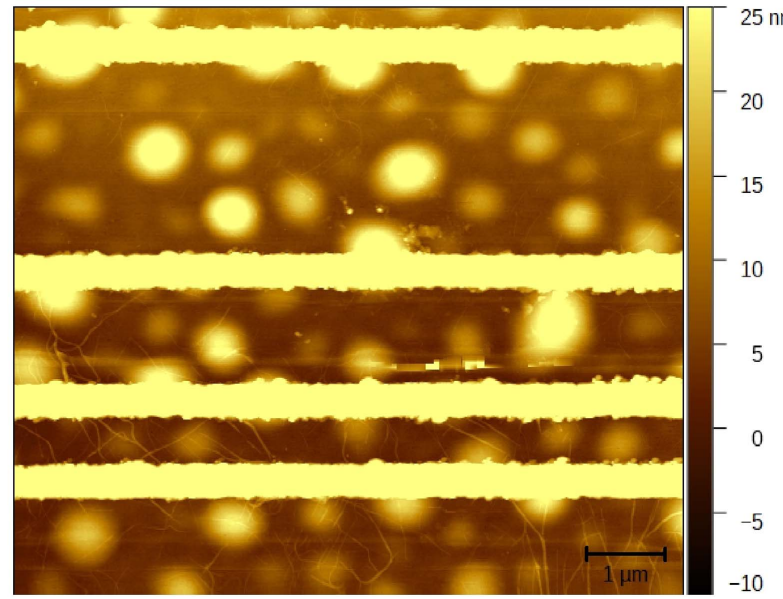

Figure 1. Dark-field optical images of the sample with SWCNTs deposited on the $\mathrm{Si} / \mathrm{SiO}_{2}$ substrate pre-implanted with $\mathrm{H}^{+}$and $\mathrm{He}^{+}$ions then clamped by metallic stripes (a) before and (b) after thermal annealing. Blisters could be seen as bright circles. (c) SEM image of the sample after thermal annealing. Blisters could be seen as dark contrast circles. Zone 'A' indicates non-clamped SWCNTs, Zone 'B' indicates SWCNTs clamped between metallic stripes (d) AFM topography image of the sample after thermal annealing.
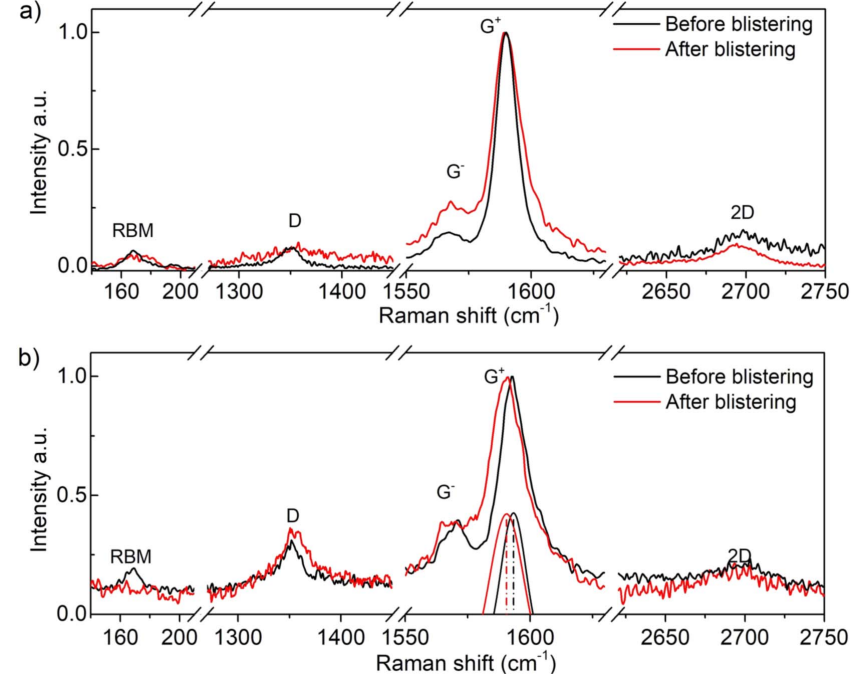

Figure 2. Representative resonant Raman spectra of SWCNTs (a) nonclamped (zone 'A' on figure 1(c)) (b) clamped between metallic stripes (zone 'B' on figure 1(c)) before (black) and after (red) blistering of the surface. Spectra are normalized by the intensity of the $\mathrm{G}^{+}$peak. On the bottom panel (b) Lorentzian fittings of $\mathrm{G}^{+}$peaks are shown, fittings are down-shifted for clarity. Excitation wavelength $\lambda=473 \mathrm{~nm}$.

Figure 2(b) shows the Raman spectra obtained on the semiconducting SWCNTs clamped at both edges between the closest pair of metallic stripes (zone B in figure 1(c)) before (black line) and after (red line) blistering of the wafer surface. In contrast to non-clamped SWCNTs, the G and 2D bands were red-shifted and the RBM band disappeared after blistering which are the hallmarks of a tensile strain in SWCNTs [36]. The absence of the RBM band in the spectra obtained on uniaxially strained SWCNTs was reported previously [33] and explained by a shift of the transition energy out of the resonance. This effect is strongly dependent on the nanotube's diameter. In the present work, the vanishing of the RBM signal was not always observed which is attributed to the contributions of several SWCNTs of different diameters probed simultaneously by a $1 \mu \mathrm{m}$ large laser spot.

The measurement of the position shift of the sharp an intense $\mathrm{G}^{+}$peak provides the most accurate estimation of strain in the SWCNT taking into account its diameter [37]. For the SWCNTs studied here, the $\mathrm{G}^{+}$peak position shift to strain ratio was estimated based on works of Cronin et al [33] and Gao et al [37]. Cronin et al [33] measured $\mathrm{G}^{+}$peak shifts in 13 different semiconducting CNTs with a diameter ranging from 1.3 to $1.5 \mathrm{~nm}$ subjected to uniaxial strain induced by AFM tip. According to these data, the mean value of $\mathrm{G}^{+}$shift rate is $17.5 \mathrm{~cm}^{-1}$ per $1 \%$ for the applied strain ranging from $0.06 \%$ to $0.53 \%$. Gao et al [37] measured the $\mathrm{G}^{+}$shift in six CNTs of well-defined diameter and chirality angle as a function of uniaxial strain. They found that shift rate increases when CNTs diameter increases and chiral angle decreases. 

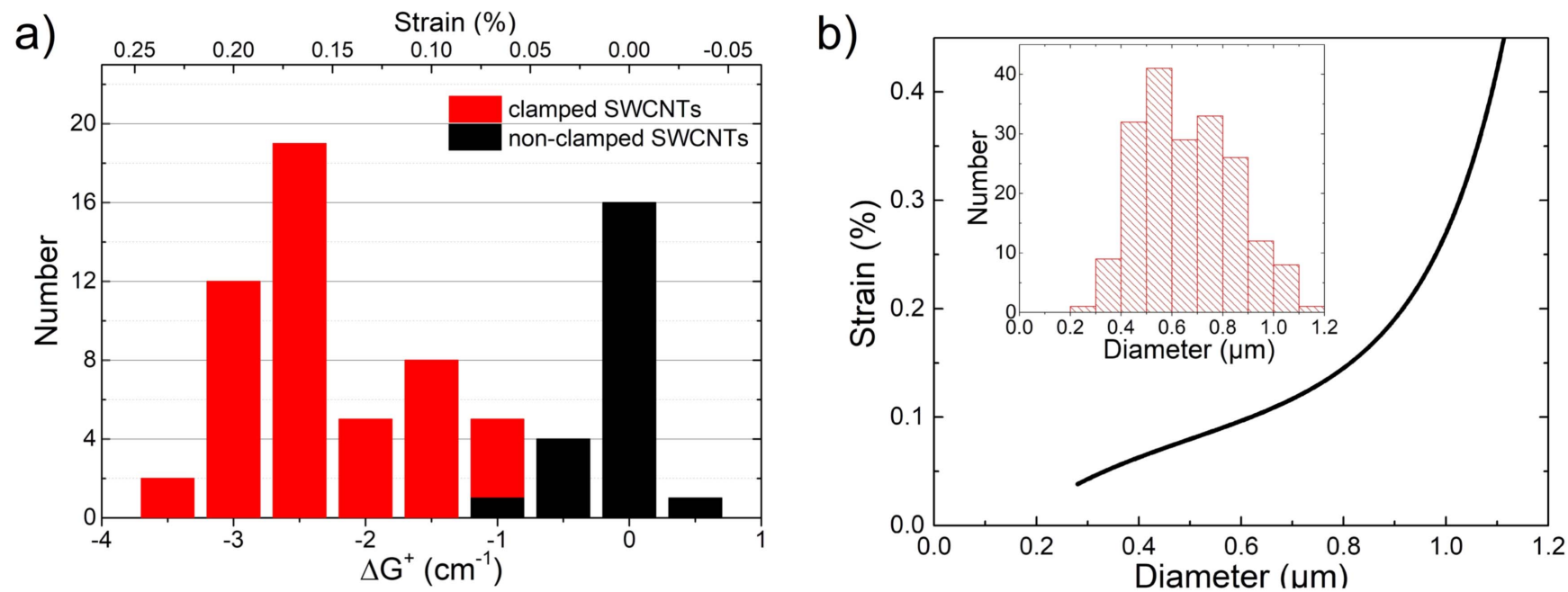

Figure 3. (a) Histogram of $\mathrm{G}^{+}$Raman peak position shifts $\Delta \mathrm{G}^{+}$measured for double-end clamped (red bars) and one end or non-clamped (black bars) SWCNTs. A negative value of $\Delta \mathrm{G}^{+}$corresponds to a red shift. (b) Strain in double-end clamped SWCNTs calculated as a function of experimentally observed range of blisters' diameters. Insert: histogram of the blister diameter distribution extracted from [24].

Based on these data, a linear approximation of $\mathrm{G}^{+}$shift rate as a function of CNT diameter gives $12 \mathrm{~cm}^{-1}$ per $1 \%$ for CNT of the diameter $1.5 \mathrm{~nm}$, which is the mean diameter of CNTs used in our experiment. Thus, in current work we used a value of $15 \mathrm{~cm}^{-1} / \%$ as a fair estimation mediated between values of the discussed articles. Therefore, for the spectrum shown in figure 2(b), the red shift $\Delta \mathrm{G}^{+}$of $2.4 \mathrm{~cm}^{-1}$ corresponds to the tensile strain of $\sim 0.16 \%$.

By applying the statistical analysis of more than 70 Raman spectra taken in different areas of the blistered sample, we have extracted the $\Delta \mathrm{G}^{+}$position shifts distribution corresponding to double-end clamped (red bars) and non (or partially)-clamped (black bars) SWCNTs (figure 3(a)). The average value of $\Delta \mathrm{G}^{+}$for double-end clamped SWCNTs being equal to $\Delta \mathrm{G}^{+}=2.3 \pm 0.5 \mathrm{~cm}^{-1}$, we evaluate the average strain within such SWCNTs of $0.15 \pm 0.03 \%$.

In the following, we estimate theoretically strain in a SWCNT which passes through the apex of a blister and is double-end clamped at the blister's base. For this, we define strain in the SWCNT as $\varepsilon(d, h)=\frac{\Delta L}{d}=\frac{L(d, h)-d}{d}$ where $d$ is the length of the SWCNT on the flat surface equal to the diameter of the blister and $L(d, h)$ is the length of the SWCNT which it obtains while passing through the apex of the blister with the height of $h$. The $L(d, h)$ is given by:

$$
L(d, h)=2 \cdot \int_{0}^{d 2} \sqrt{1+\left(\frac{\partial \Delta z(r)}{\partial r}\right)^{2}} d r
$$

where $\Delta z(r)$ is the blister surface displacement in the vertical $z$ direction expressed as a function of the in-plane radial distance $r$. For a blister approximated by a circular plate which is clamped at the edge under uniform pressure loading [38], the $\Delta z(r)$ is given by:

$$
\Delta z=h\left(1-4 \frac{r^{2}}{d^{2}}\right)^{2}
$$

There is the one-to-one relation between blister diameters and blister heights for the given implantation and annealing conditions as it was shown in [24] which can be fitted by $h(d)=\left(-213+8 \times 10^{-6} \exp ((d+1.55) / 0.178)+179 \exp \right.$ $((d+1.55) / 9.676)) \times 10^{-3}$. Finally, by substituting this expression in equations (2) and (1), we calculated strain in the SWCNT as a function of experimentally observed range of blisters' diameters and the result is shown in figure 3(b). This figure shows that strain in SWCNT rapidly increases from $0.04 \%$ to $0.45 \%$ when the diameter of blisters increases from 0.3 to $1.1 \mu \mathrm{m}$ which is well consistent with the rapid increase of a blister height. For the given blisters diameter distribution shown in insert of figure 3(b), a blister with the average diameter of $0.67 \mu \mathrm{m}$ and the height of $15 \mathrm{~nm}$ would provide tensile strain of $0.11 \%$ in a SWCNT clamped over its edge. This value is in a good agreement with that obtained experimentally by using resonant Raman spectroscopy. Thus, strain induced by blistering was fully transferred to SWCNTs double-end clamped by the metal stripes spaced by a distance close to the average diameter of the blisters.

In order to be able to manipulate the strain in the SWCNTs that pass over the blisters, one should be able to fabricate blisters of predefined diameters and heights. The analysis of the previously obtained data on the blisters' dimensions, as a function of implantation conditions [21-32], evidences that the height-todiameter ratio of a blister can be adjusted by choosing an appropriate $\mathrm{H}$ ions implantation energy that assigns the crack depth position. The closer the crack to the wafer surface, the higher is the corresponding blister. However, the average diameter of blisters decreases when the cracks are formed closer to the wafer surface. Such effect can be compensated by adjusting total $\mathrm{H}$ and $\mathrm{He}$ fluences and $\mathrm{He}$ to $\mathrm{H}$ fluences ratio. In combination with the appropriate annealing conditions, it would be possible to manipulate the density and thus, to maximize the surface fraction of blisters of required average diameter and height-to-diameter ratio. Finally, the blisters eventual parameters can be homogenized by applying a periodic stencil mask prior to 
ion implantation which would allow their lateral ordering, the site-specific and size-specific formation within the mask openings.

\section{Conclusion}

In this work, we have proposed the novel method of strain transfer to surface supported low-dimensional nanomaterials. The method consists of several steps: (i) $\mathrm{H}^{+}$and $\mathrm{He}^{+}$sequential ion implantation in a wafer; (ii) transfer of $1 \mathrm{D}$ or $2 \mathrm{D}$ material onto the wafer surface; (iii) nanostructuring of the material and metal pattern deposition; (iv) thermal annealing resulting in the surface blistering. We have applied this method to SWCNTs deposited on a $\mathrm{SiO}_{2} / \mathrm{Si}(001)$ substrate. By using Raman spectroscopy measurements, we have demonstrated an effective bottom-up strain transfer to SWCNTs by blisters provided the nanotubes were double-end clamped by metal stripes, which were spaced by a distance close to the average diameter of the blisters. We have evaluated the tensile strain within such SWCNTs of $0.15 \pm 0.03 \%$ which was found to be in good agreement with the value of tensile strain estimated from the average blister diameter and associated height.

We believe that the application of this method can be further extended to other $1 \mathrm{D}$ and 2D materials. All nanostructuring of the materials can be processed on a flat substrate and blistering can be programmed as a last step of the fabrication. The morphology of blisters can be controlled by varying implantation and annealing conditions. By applying a periodic stencil mask prior to ion implantation, the lateral ordering, the site-specific and size-specific formation of blisters within the mask openings can be obtained after thermal annealing, leaving the rest of the surface area flat. Finally, such possibility of a controlled, repetitive and desired manipulation of strain within nanostructures and, thus, of their electronic band structure opens a way for potential application of this method in a large-scale production.

\section{Acknowledgments}

We thank Dr G Deligeorgis for useful discussions and comments, as well as the Institute of Electronic Structure and Laser of the Foundation for Research and Technology-Hellas (IESL-FORTH) and Crete Center for Quantum Complexity and Nanotechnology (CCQCN). We thank B Pecassou for ion implantation. VP, KK and MM thank the Russian Foundation for Basic Research (RFBR), according to the research project no. 16 32-60081 mol_a_dk, for financial support.

\section{ORCID iDs}

V S Prudkovskiy (ib https://orcid.org/0000-0003-4375-5561

K P Katin (1) https://orcid.org/0000-0003-0225-5712

M M Maslov (iD https://orcid.org/0000-0001-8498-4817

N Cherkashin (1) https://orcid.org/0000-0002-0322-0864

\section{References}

[1] Morozov S V, Novoselov K S, Katsnelson M I, Schedin F, Elias D C, Jaszczak J A and Geim A K 2008 Giant intrinsic carrier mobilities in graphene and its bilayer Phys. Rev. Lett. 100016602

[2] Bolotin K I, Sikes K J, Jiang Z, Klima M, Fudenberg G, Hone J, Kim P and Stormer H L 2008 Ultrahigh electron mobility in suspended graphene Solid State Commun. 146 $351-5$

[3] Baringhaus $\mathrm{J}$ et al 2014 Exceptional ballistic transport in epitaxial graphene nanoribbons Nature 506 349-54

[4] Pospischil A, Humer M, Furchi M M, Bachmann D, Guider R, Fromherz T and Mueller T 2013 CMOS-compatible graphene photodetector covering all optical communication bands Nat. Photon. 7 892-6

[5] Dragoman M, Deligeorgis G, Muller A, Cismaru A, Neculoiu D, Konstantinidis G, Dragoman D, Dinescu A and Comanescu F 2012 Millimeterwave Schottky diode on graphene monolayer via asymmetric metal contacts J. Appl. Phys. 112084302

[6] Deligeorgis G, Coccetti F, Konstantinidis G and Plana R 2012 Radio frequency signal detection by ballistic transport in Y-shaped graphene nanoribbons Appl. Phys. Lett. 101 013502

[7] Dragoman M, Neculoiu D, Cismaru A, Muller A A, Deligeorgis G, Konstantinidis G, Dragoman D and Plana R 2011 Coplanar waveguide on graphene in the range 40 MHz-110 GHz Appl. Phys. Lett. 99033112

[8] Saito R, Fujita M, Dresselhaus G and Dresselhaus M S 1992 Electronic structure of chiral graphene tubules Appl. Phys. Lett. 60 2204-6

[9] Novoselov K S, Geim A K, Morozov S V, Jiang D, Zhang Y, Dubonos S V, Grigorieva I V and Firsov A A 2004 Electric field effect in atomically thin carbon films Science $\mathbf{3 0 6}$ 666-9

[10] Krupke R, Hennrich F, Lohneysen H and Kappes M M 2003 Separation of metallic from semiconducting single-walled carbon nanotubes Science 301 344-7

[11] Hersam M C 2008 Progress towards monodisperse singlewalled carbon nanotubes Nat. Nanotechnol. 3 387-94

[12] Arnold M S, Stupp S I and Hersam M C 2005 Enrichment of single-walled carbon nanotubes by diameter in density gradients Nano Lett. 5 713-8

[13] Arnold M S, Green A A, Hulvat J F, Stupp S I and Hersam M C 2006 Sorting carbon nanotubes by electronic structure using density differentiation Nat. Nanotechnol. 1 $60-5$

[14] Green A A and Hersam M C 2008 Colored semitransparent conductive coatings consisting of monodisperse metallic single-walled carbon nanotubes Nano Lett. 8 1417-22

[15] Block B, Antaris A L, Seo J T, Green A A and Hersam M C 2010 Sorting single-walled carbon ACS Nano 4 4725-32

[16] Yang L, Anantram M P, Han J and Lu J P 1999 Band-gap change of carbon nanotubes: effect of small uniaxial and torsional strain Phys. Rev. B 60 13874-8

[17] Ni Z H, Chen W, Fan X F, Kuo J L, Yu T, Wee A T S and Shen Z X 2008 Raman spectroscopy of epitaxial graphene on a SiC substrate Phys. Rev. B 77115416

[18] Pereira V M, Castro Neto A H and Peres N M R 2009 Tightbinding approach to uniaxial strain in graphene Phys. Rev. B 80045401

[19] Cocco G, Cadelano E and Colombo L 2010 Gap opening in graphene by shear strain Phys. Rev. B 81241412

[20] Kwon S, Choi S, Chung H J, Yang H, Seo S, Jhi S H and Young Park J 2011 Probing nanoscale conductance of monolayer graphene under pressure Appl. Phys. Lett. 99 013110 
[21] Evans J H 1976 A mechanism of surface blistering on metals irradiated with helium ions $\mathrm{J}$. Nucl. Mater. 61 1-7

[22] Qian C and Terreault B 2001 Blistering of silicon crystals by low keV hydrogen and helium ions J. Appl. Phys. 90 5152-8

[23] Personnic S, Bourdelle K K, Letertre F, Tauzin A, Cherkashin N, Claverie A, Fortunier R and Klocker H 2008 Impact of the transient formation of molecular hydrogen on the microcrack nucleation and evolution in $\mathrm{H}$-implanted $\mathrm{Si}$ (001) J. Appl. Phys. 103 1-9

[24] Cherkashin N, Daghbouj N, Darras F X, Fnaiech M and Claverie A 2015 Cracks and blisters formed close to a silicon wafer surface by $\mathrm{He}-\mathrm{H}$ co-implantation at low energy J. Appl. Phys. 118245301

[25] Grisolia J, Ben Assayag G, Claverie A, Aspar B, Lagahe C and Laanab L 2000 A transmission electron microscopy quantitative study of the growth kinetics of $\mathrm{H}$ platelets in $\mathrm{Si}$ Appl. Phys. Lett. 76852

[26] Hebras X, Nguyen P, Bourdelle K K, Letertre F, Cherkashin N and Claverie A 2007 Comparison of platelet formation in hydrogen and helium-implanted silicon Nucl. Instrum. Methods Phys. Res. B 262 24-8

[27] Moutanabbir O, Terreault B, Chicoine M, Schiettekatte F and Simpson P J 2007 Influence of isotopic substitution and He coimplantation on defect complexes and voids induced by $\mathrm{H}$ ions in silicon Phys. Rev. B 75 1-11

[28] Terreault B 2007 Hydrogen blistering of silicon: progress in fundamental understanding Phys. Status Solidi a 204 2129-84

[29] Agarwal A, Haynes T E, Venezia V C, Holland O W and Eaglesham D J 1998 Efficient production of silicon-on-insulator films by co-implantation of $\mathrm{He}^{+}$with $\mathrm{H}^{+}$Appl. Phys. Lett. 72 1086-8
[30] Daghbouj N, Cherkashin N and Claverie A 2018 A method to determine the pressure and densities of gas stored in blisters: application to $\mathrm{H}$ and $\mathrm{He}$ sequential ion implantation in silicon Microelectron. Eng. 190 54-6

[31] Cherkashin N, Daghbouj N, Seine G and Claverie A 2018 Impact of $\mathrm{He}$ and $\mathrm{H}$ relative depth distributions on the result of sequential $\mathrm{He}^{+}$and $\mathrm{H}^{+}$ion implantation and annealing in silicon J. Appl. Phys. 123 1-9

[32] Daghbouj N, Cherkashin N, Darras F X, Paillard V, Fnaiech M and Claverie A 2016 Effect of the order of $\mathrm{He}^{+}$ and $\mathrm{H}^{+}$ion co-implantation on damage generation and thermal evolution of complexes, platelets, and blisters in silicon J. Appl. Phys. 119 1-8

[33] Cronin S B, Swan A K, Ünlü M S, Goldberg B B, Dresselhaus M S and Tinkham M 2005 Resonant Raman spectroscopy of individual metallic and semiconducting single-wall carbon nanotubes under uniaxial strain Phys. Rev. B 72035425

[34] Dresselhaus M S, Dresselhaus G, Saito R and Jorio A 2005 Raman spectroscopy of carbon nanotubes Phys. Rep. 409 47-99

[35] Li T, Ayari A and Bellon L 2015 Adhesion energy of single wall carbon nanotube loops on various substrates $J$. Appl. Phys. 117164309

[36] Qiu W, Li Q, Lei Z-K, Qin Q-H, Deng W-L and Kang Y-L 2013 The use of a carbon nanotube sensor for measuring strain by micro-Raman spectroscopy Carbon 53 161-8

[37] Gao B, Jiang L, Ling X, Zhang J and Liu Z 2008 Chiralitydependent Raman frequency variation of single-walled carbon nanotubes under uniaxial strain J. Phys. Chem. C 112 20123-5

[38] Timoshenko S and Woinowsky-Krieger S 1959 Theory of Plates and Shells ed R H Phelps (New York: McGraw-Hill) 